\title{
First Masquerading as Gallstones, Pulmonary Hypertension Mimics PE
}

\author{
John Quinn'1,2, Sajana Ukwatta1, Chris Luke³, Tomas Zeleny4, Vladimir Bencko5 \\ ${ }^{1}$ Mercy University Hospital, Cork, Ireland \\ ${ }^{2}$ Prague Center for Global Health, Institute of Hygiene and Epidemiology, First Faculty of Medicine, Charles \\ University in Prague, Prague, Czech Republic \\ ${ }^{3}$ Cork University Hospital, Mercy University Hospital, Cork, Ireland \\ ${ }^{4}$ Institute of Economic Studies, Charles University in Prague, Prague, Czech Republic \\ ${ }^{5}$ Institute of Hygiene and Epidemiology, First Faculty of Medicine, Charles University in Prague, Prague, Czech \\ Republic \\ Email: John.quinn@If1.cuni.cz, Sajana112@hotmail.com, cluke@muh.ie, Zeleny.t@gmail.com, \\ Vladimir.bencko@If1.cuni.cz
}

Received 3 January 2015; accepted 25 December 2015; published 28 December 2015

Copyright (C) 2015 by authors and Scientific Research Publishing Inc.

This work is licensed under the Creative Commons Attribution International License (CC BY).

http://creativecommons.org/licenses/by/4.0/

(c) (i) Open Access

\section{Abstract}

Patients presenting to emergency and urgent care centers with calf pain after long and short-haul flights are a common presentation throughout Europe. Patients fitting an epidemiological risk profile for cholelithiasis and presenting with right upper quadrant abdominal pain can also be a common presentation fitting of a specific patient profile. However, pulmonary hypertension can present in a nuanced and possible missed chronic and acute presentation. The patient case we present profiles a mildly obese 54-year-old Caucasian woman and recent holiday maker with unilateral calf pain and shortness of breath after traveling on a long-haul flight with tertiary symptoms of indigestion and epigastric discomfort indicative of gastroenteritis. This case highlights the required diligence for emergency physicians to maintain a high index of suspicion and broad differential diagnosis in the undifferentiated patient with seemingly common or classic presentations. We find that a serendipitous definitive diagnosis is made by following a systematic and organized approach.

\section{Keywords}

Venouse Thromboembolism (DVT), Cholylithisis, Pulmonary Hypertension, Emergency Medicine Assessment

\section{Introduction}

A period of prolonged seated immobility is recognized as one of the major risk factors for developing deep vein

How to cite this paper: Quinn, J., Ukwatta, S., Luke, C., Zeleny, T. and Bencko, V. (2015) First Masquerading as Gallstones, Pulmonary Hypertension Mimics PE. Case Reports in Clinical Medicine, 4, 376-380.

http://dx.doi.org/10.4236/crcm.2015.412076 
thrombus (DVT) and pulmonary embolism (PE). PE can present with acute shortness of breath, but also be masked by underlying disease or the occult presentation. Long-distance air travel and prolonged sitting in relation to work or recreation have been shown to increase the risk of DVT [1]-[3]. Risk factors for cholyslithiasis, or gall bladder stones, are increased for women with mild to moderate increased body mass index (BMI), alcohol consumption, smoking and at times of ingestion of highly fatty foods [4]. Definitive diagnosis and treatment for pulmonary hypertension in the absence of significant disease or risk factors is an easily missed pathology in the emergency department setting. Patients with pulmonary hypertension (PAH) who do not receive early diagnosis and disease-targeted therapy have a poor quality of life (QoL) and high mortality at rates [5]. Clearly, diagnosing early those patients with early or moderate pulmonary hypertension reduces morbidity and mortality.

The clinical skills, biochemical and hematological diagnostic criteria for PE, gall stones and pulmonary hypertension are not a commonplace. Presentations for these pathologies may be more nuanced and dynamic across patient populations than conventional approach would abide. The emergency physician must remain diligent in their workup of all patients, regardless of how simple or easy the presentation chief complaint may at first seem.

\section{Case Presentation}

\subsection{History of Present Illness}

A 54-year-old holiday maker presents to the emergency department (ED) in the afternoon after a transatlantic flight with unilateral calf pain, shortness of breath and mild indigestion with abdominal pain at 9/10 on the numeric pain rating pain scale. She denied chest paid and denied nausea and vomiting. Her vital signs at triage are listed in Table 1.

The patient is a 54-year-old women of modest BMI exiting a plane from Lanzorate Spain complaining of right lower calf pain and sudden on set of shortness of breath that morning. The head, eyes, earns, nose and throat (HEENT) exam is unremarkable, chest exam exhibits clear and equal lung sounds with coordinating S1 and S2 cardiac sounds. No gallops, murmurs or extra sounds heard. Abdominal exam is remarkable for mild right upper quadrant (RUQ) pain, increasing with inspiration with the rest of the abdomen being soft and non-tender, nondistended. Bowel sounds are heard. Upper and lower extremity exam exhibits equal pulses in both arms and legs.

\subsection{Further Exam}

Calves are equal in circumference and the affected calf is not hot or warm to touch, there are no collateral nonvericose superficial veins present, there is no pitting edema, mild tenderness to affected limb with pulse, motor and sensory intact distally and equal bilaterally. Further exam is unremarkable. The patient is not in acute respiratory distress but is moderately agitated with discomfort and pain to calf and abdomen. Bloods are taken during time of clinical assessment to include cardiac markers (Troponin T), D-Dimer, liver function tests (LFTs), basic coagulation studies, renal profile and full blood count (FBC). A chest X-ray and ECG, ultrasound gall bladder and CT abdomen and urine dip analysis are all ordered. The patient is offered pain management to include a proton pump inhibitor (PPI), paracetamol, normal saline fluid rehydration therapy and antiemetic are administered.

\begin{tabular}{|c|c|}
\hline Temperature & $37.4 \mathrm{C}$ \\
\hline Pulse & 91 bpm \\
\hline Blood pressure & $174 / 112$ \\
\hline Oxygen saturation & $99 \%$ on room air \\
\hline Respiratory rate & 22 per minute \\
\hline Pain & $9 / 10$ (10 is the worst possible pain, zero is no pain at all) \\
\hline
\end{tabular}




\subsection{Working Diagnosis}

The working diagnosis is DVT and or cholecystitis with PE and or cardiac cause being tertiary and at the bottom of the differential diagnosis. No obvious infectious or septic signs or symptoms are encountered and this is not a defining feature or element of the clinical impression.

\section{Findings}

The ECG was normal, the chest X-ray unremarkable. D-Dimer’s were elevated, LFTs were all elevated, an unremarkable ultrasound study for DVT and secondary CT investigation for cholycystitis revealed elements of latent pulmonary hypertension in a low risk patient.

\section{Discussion}

The pathophysiology of venous thrombosis involves venous stasis, hypercoagulability, and endothelial dysfunction. This description was termed Virchow's triad, described initially in 1856 by the German pathologist Ruldoph Virchow [6]. DVT is a non-communicable disease (NCD). The association between prolonged sitting and venous thromboembolism (VTE) leading to lethal pulmonary embolism was first recognized by Simpson during the London Blitz in World War II, when he reported deaths from pulmonary embolism in people spending hours sitting in chairs in air-raid shelters [7]. DVT remains a major threat to sudden death and increased morbidity and mortality globally for exceedingly sedentary lifestyles, work habits and less physically demanding work requirements in service economies.

D-dimers are a hematology based blood test that detects the cross-linked fibrin degradation fragment. Elevations in this fragment are seen in primary and secondary fibrinolysis; during thrombolytic or defibrination therapy with tissue plasminogen activator; as a result of thrombotic disease, such as deep-vein thrombosis, pulmonary embolism or disseminated intravascular coagulation (DIC); in vasoocclusive crisis of sickle cell anemia; in malignancies; and in prolonged surgical interventions and repair.

The clinical features of DVT, such as localized swelling, redness, tenderness, and peripheral edema, are nonspecific, and the diagnosis should always be confirmed by objective tests; please see Wells Score in Table 2 below. As this case illustrates, many ambulatory patients with clinically suspected DVT may have another cause for their symptoms.

Venous ultrasonography (US) is the noninvasive imaging method of choice for diagnosing DVT. US is highly accurate for diagnosis of proximal vein thrombosis, with a sensitivity and specificity approaching 95\% [8]. The sensitivity for symptomatic calf vein thrombosis is considerably lower and appears to be highly operator dependent. For this reason, many centers do not examine the deep veins of the calf with ultrasonography; instead,

Table 2. Wells Model for Determining Clinical Suspicion of Deep Vein Thrombosis (DVT) (Pretest probability of DVT is calculated from the total points: $>2$ points, high; 1 or 2 , moderate; $<1$, low).

\begin{tabular}{l}
\hline \multicolumn{1}{c}{ Variable } \\
\hline Active cancer (treatment ongoing or within previous 6 months or palliative) \\
Paralysis, paresis, or recent plaster immobilization of the lower extremities \\
Recently bedridden >3 days or major surgery within 4 weeks \\
Localized tenderness along the distribution of the deep venous system \\
Entire leg swollen \\
Calf swelling 3 cm greater than on asymptomatic side (measured 10 cm below tibial tuberosity) \\
Pitting edema confined to the symptomatic leg \\
Dilated superficial veins (non-varicose) \\
Alternative diagnosis as likely or greater than that of DVT \\
Total Score:
\end{tabular}


if examination of the proximal veins excludes proximal DVT in a patient with a moderate or high clinical assessment for DVT, the test is repeated in 7 days to detect the small number of calf vein thrombi $(\sim 3 \%)$ that subsequently extend into the proximal veins and if the test remains negative after 7 days, the risk that thrombus is present and will extend to the proximal veins is negligible, and it is safe to withhold treatment [8]. Some centers in Ireland and the UK will start DVT therapy empirically within 12 to 24 hours of onset of signs and symptoms.

Furthermore, cholelithiasis are as well a NCD threat growing in prevalence in the moderate to most developed nations and can be costly to overburdened healthcare systems in developed countries. The prevalence of gallstones appears to be rising annually across North America and Europe, with many being "silent," and approximately one third eventually causing symptoms that warrant investigations and possible complications such as cholecystitis [9]. Definitive diagnostic criteria for gall stones includes the clinical presentation of right upper quadrant (RUG) pain, sometimes radiating to the right shoulder in acute presentations, visceral epigastric painmoderate to severe in RUQ and possibly with associated nausea and vomiting and physical findings of fever (rare), a positive Murphy's sign (Right subcostal tenderness with inspiratory arrest), possibly a palpable gall bladder on abdominal exam and mild jaundice. Laboratory studies include white cells, leukocytes with band forms increased, serum bilirubin raised with elevated alkaline phosphatase and aminotransferase in the absence of bile duct stone or liver infection, serum amylase and lipase levels elevated in the absence of pancreatitis are also indicative of gall bladder disease.

Imaging studies for cholelithiasis remain the gold standard in definitive diagnosis for both cholylithiasis and cholycytitis. Specifically, ultrasound, hepatobiliary scintigraphy and abdominal commuted tomography (CT) are best practice gold standard definitive diagnostic tools. Of course, treatment for cholysitits can be conservative to interventional, with cholecystectomy offering definitive treatment.

Lastly, primary pulmonary arterial hypertension $(\mathrm{PPH})$ is a rare disease characterized by elevated pulmonary artery pressure with no apparent cause [10]. Blood flow to tissues is dependent on a gradient between an area of higher pressure (mean arterial pressure, MAP) and the lower-pressure side of the venous system (represented by a central venous pressure, CVP). The mean arterial pressure may fall if the cardiac output (CO) is reduced or if the total peripheral resistance (TPR) in the arterial tree falls; mean arterial pressure (MAP) $=\mathrm{CO} \times \mathrm{TPR}$.

PPH is also termed pre-capillary pulmonary hypertension or, as is currently preferred, idiopathic pulmonary arterial hypertension (IPAH). Pulmonary hypertension can be numerically defined as mean pulmonary artery pressure (MAP) $>25 \mathrm{~mm} \mathrm{Hg}$ at rest or $>30 \mathrm{~mm} \mathrm{Hg}$ with exercise. This case illustrates primary pulmonary arterial hypertension (PPH), which is more common in women as there is a genetic predisposition of mutations in genes associated with transforming growth factor- $\beta$. This leads to vascular hyperactivity with proliferation of smooth muscle. This is in contrast to secondary pulmonary hypertension, which leads to endothelial cell dysfunction, and a loss of vasodilators and an increase in vasoconstrictors, which leads to hypoxemia and respiratory acidosis, stimulate vasoconstriction of pulmonary arteries.

Clinical findings of pulmonary hypertension can be exertional dyspnea, chest pain and a chest X-ray showing a tapering of the pulmonary arteries. Clinical exam findings can demonstrate accentuated $\mathrm{P}_{2}$ (sign of $\mathrm{PH}$ ) with left parasternal heave (sign of right ventricular hypertrophy). Pulmonary hypertension imposes an increased afterload on the right ventricle leading to right-sided heart failure due to cor pulmonale. Diagnosis is definitive through catheterization to measure pressures and transthoracic Doppler echocardiogram can offer non-invasive testing; CT chest can also find quantitiative enlargement of the main pulmonary arteries with rapid tapering of distal vessels and marked or slight right ventricular enlargement. If left untreated or not diagnosed early, IPAH leads to right-sided heart failure and death.

\section{Conclusions}

Core concepts and departmental standardized diagnostic criteria for seemingly disparate chief complaints of unilateral calf pain with shortness of breath in periods of increased immobilization; colicky right upper quadrant pain that is Murphy's positive in epidemiologically high risk populations and occult findings of pulmonary hypertension should be reviewed to minimize missed diagnosis and to promote best medical outcomes. As always in emergency medicine, diagnostic tests, biochemically, hematologicaly and clinically, must not be viewed with tunnel vision but through an interdisciplinary approach for all undifferentiated patients.

Patients presenting to EDs with textbook histories, classic signs and symptoms of DVT must be approached with an open mind and diagnosis fixation should be avoided. Furthermore, the epidemiological profiles for cholylithiasis should also be approached systemically with diagnostics interpreted through an interdisciplinary lens. 
This case report describes the required diligence for emergency physicians to maintain a high index of suspicion and broad differential diagnosis in the undifferentiated patient with seemingly common or classic presentations so as not miss treatable conditions.

\section{Acknowledgements}

The authors acknowledge their thanks to the 1st Faculty of Medicine, Charles University in Prague and to the Institute of Economic Studies at Faculty of Social Sciences of Charles University in Prague for their support of our study by the grants GAUK 910892 and PRVOUK- P28/1LF/6.

\section{Competing Interests}

The authors declare that they have no competing interests.

\section{Authors' Contributions}

JQ drafted the manuscript; SU added details of patient care, presentation and overall management of the case presentation. CL and SU provided editing and final approval of the manuscript.

\section{References}

[1] Cruickshank, J.M., Gorlin, R. and Jennett, B. (1988) Air Travel and Thrombotic Episodes: The Economy Class Syndrome. Lancet, 2, 497-498. http://dx.doi.org/10.1016/S0140-6736(88)90134-1

[2] Beasley, R., Heuser, P. and Raymond, N. (2005) SIT (Seated Immobility Thromboembolism) Syndrome: A 21st Century Lifestyle Hazard. The New Zealand Medical Journal, 118, U1376.

[3] Chang, H.-C.L., Burbridge, H. and Wong, C. (2013) Extensive Deep Vein Thrombosis Following Prolonged Gaming (“Gamers Thrombosis”): A Case Report. Journal of Medical Case Reports, 7, 235. http://dx.doi.org/10.1186/1752-1947-7-235

[4] Everhart, J.E., Khare, M., Hill, M. and Maurer, K.R. (1999) Prevalence and Ethnic Differences in Gallbladder Disease in the United States. Gastroenterology, 117, 632-639. http://dx.doi.org/10.1016/S0016-5085(99)70456-7

[5] National Pulmonary Hypertension Centres of the UK and Ireland (2008) Consensus Statement on the Management of Pulmonary Hypertension in Clinical Practice in the UK and Ireland. Heart, 94, i1-i41.

[6] Anning, S.T. (1957) The Historical Aspects of Venous Thrombosis. Medical History, 1, 28-37. http://dx.doi.org/10.1017/S0025727300020743

[7] Beasley, R., Raymond, N., Hill, S., Nowitz, M. and Hughes, R. (2003) eThrombosis: The 21st Century Variant of Venous Thromboembolism Associated with Immobility. European Respiratory Journal, 21, 374-376. http://dx.doi.org/10.1183/09031936.03.00039403

[8] Kearon, C. (2013) Chapter 6, the Respiratory System. In: Bope, E.T. and Kellerman, R.D., Eds., Conn's Current Therapy 2013, Saunders, 327-361.

[9] Diehl, A.K. (1991) Epidemiology and Natural History of Gallstone Disease. Gastroenterology Clinics of North America, 20, 1-19.

[10] McLaughlin, V.V. and McGoon, M.D. (2006) Pulmonary Arterial Hypertension. Circulation, 114, 1417-1431. http://dx.doi.org/10.1161/CIRCULATIONAHA.104.503540 